Int. J. Dev. Biol. 53: 983-991 (2009)

doi: $10.1387 / \mathrm{ijdb} .072435 \mathrm{nh}$

\title{
Lasp1 misexpression influences chondrocyte differentiation in the vertebral column
}

\author{
NATASCHA HERMANN-KLEITER ${ }^{*}, 1$, NASSIM GHAFFARI-TABRIZI ${ }^{2}$, MICHAEL J.F. BLUMER $^{3}$, \\ CHRISTOPH SCHWARZER ${ }^{4}$, MAGDALENA A. MAZUR ${ }^{5}$ and ISABELLA ARTNER ${ }^{*}, 5$
}

\begin{abstract}
${ }^{1}$ Dept. Medical Genetics, Molecular and Clinical Pharmacology Medical University, Innsbruck, Austria, ${ }^{2}$ Institute of Pathophysiology and Immunology, Centre of Molecular Medicine, Medical University of Graz, Austria, ${ }^{3}$ Dept. Anatomy, Histology and Embryology, Division of Clinical and Functional Anatomy, Innsbruck Medical University, Innsbruck, Austria, ${ }^{4}$ Dept. Pharmacology, Innsbruck Medical University, Innsbruck, Austria and ${ }^{5}$ Developmental Biology Program, Stem Cell Center, Lund University, Sweden
\end{abstract}

\begin{abstract}
The mouse mutant wavy tail Tg(Col1a1-lacZ)304ng was created through transgene insertion and exhibits defects of the vertebral column. Homozygous mutant animals have compressed tail vertebrae and wedge-shaped intervertebral discs, resulting in a meandering tail. Delayed closure of lumbar neural arches and lack of processus spinosi have been observed; these defects become most prominent during the transition from cartilage to bone. The spina bifida was resistant to folic acid treatment, while retinoic acid administration caused severe skeletal defects in the mutant, but none in wild type control animals. The transgene integrated at chromosome 11 band $D$, in an area of high gene density. The insertion site was located between the transcription start sites of the Rp/23 and Lasp1 genes. LASP1 (an actin binding protein involved in cell migration and survival) was found to be produced in resting and hypertrophic chondrocytes in the vertebrae. In mutant vertebrae, temporal and spatial misexpression of Lasp 1 was observed, indicating that alterations in Lasp 1 transcription are most likely responsible for the observed phenotype. These data reveal a yet unappreciated role of Lasp1 in chondrocyte differentiation during cartilage to bone transition.
\end{abstract}

KEY WORDS: transgene insertion, cartilage bone transition, folic acid, retinoic acid, collagen

\section{Introduction}

Malformations of the vertebral column, like scoliosis, skyphosis, or spina bifida, are frequent congenital disorders in human. However, the precise genetic basis underlying these defects is known only in surprisingly few instances, and many disorders are multifactorial, involving either multiple genes or gene/enviromental interactions (Helwig et al., 1995). The mouse provides a useful model for human congenital defects, in particular of the skeleton. Over 190 spontaneous mouse mutants exhibiting a skeletal phenotype are known (reviewed in Kibar etal., 2007; Searle etal., 1989), and the responsible genes have been identified in a number of them. Targeted mutation of specific genes facilitated the study of their function in distinct developmental processes, including bone and skeletal development. Yet, this approach does not find and identify genes previously not suspected to play a role in skeletogenesis.
Insertional mutagenesis results from the often random integration of foreign DNA sequence (e.g. a retrovirus or a transgene) within or near a gene. We previously reported (Kleiter et al., 2002) that the wavy tail $\mathrm{Tg}(\mathrm{Col1a1-lacZ)304ng} \mathrm{mouse} \mathrm{line} \mathrm{was} \mathrm{pro-}$ duced by pronuclear injection of a $15 \mathrm{~kb}$ reporter gene construct in which the Col1a1 promoter was fused to a $L a c Z$ reporter gene. Beta galactosidase staining faithfully reflected endogenous Col1a1 transcription in transgenic animals (Ghaffari-Tabrizi N., in preparation). No other transgenic mouse line carrying this construct showed any skeletal abnormalities, indicating that transgene expression itself did not account for the skeletal deformations observed, suggesting instead that the insertion had disrupted or influenced the expression of an endogenous gene. Isolation of the

Abbreviations used in this paper: Col, collagen; ECM, extracellular matrix; Lasp, Lim and SH3 protein; RA, retinoic acid.

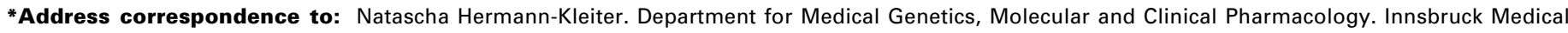

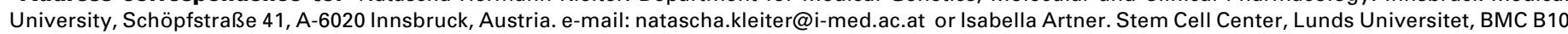
Klinikgatan 26, 22184 Lund, Sweden. e-mail: isabella.artner@med.lu.se
}

Accepted: 11 July 2008. Published online: 16 March 2009.

ISSN: Online 1696-3547, Print 0214-6282 
insertion site has been reported before (Kleiter et al., 2000), the transgene locus mapped on chromosome 11 close to the Hoxb cluster and Erbb2, where a $185 \mathrm{~kb}$ region including the insertion site was isolated and searched for transcribed regions. 6 genes were identified: Lasp1, Rpl23, MIlt6, Pip5k2b, Psmb3, Zfp144 (Kleiter et al., 2002). The insertion site was located between the transcription start sites of Lasp1 and Rp/23, with the Lasp1 transcription start site situated just 6,785 bp from the insertion site. Previous reports had demonstrated that neither Rp/23 expression nor the expression of the other genes MIlt6, Pip5k2b, Psmb3, Zfp144was perturbed in transgenic animals (Kleiter etal., 2000).

The LASP1 protein (for LIM and SH3 protein 1) consists of a $\mathrm{LIM}$, an $\mathrm{SH} 3$ domain and 2 actin binding modules. The protein was first identified in a screen for genes amplified in breast cancer (Tomasetto et al., 1995). Northern blot analysis showed that Lasp1 mRNA was transcribed in various tissues (e.g. colon, intestine, stomach, kidney, brain (Schreiber et al., 1998a)), with its expression being restricted to specific cell types in the organs investigated (e.g. gastric parietal cells within the stomach (Chew et al., 1998). The actin binding domain mediates interaction between LASP1 and actin at cell membrane extensions (Chew et al., 1998; Nakagawa et al., 2006; Schreiber et al., 1998b) and is necessary for cell migration and survival in response to growth factors and extracellular matrix (ECM) proteins (Lin et al., 2004).

So far, expression of Lasp1 has not been reported in skeletal tissues. Here we report that Lasp1 transcripts were detected in resting and hypertrophic chondrocytes during skeletal development in the wild type. Wavy tail Tg(Col1a1-lacZ)304ng mutant mice had aberrant Lasp1 expression in proliferating chondrocytes and the periosteum. Our results suggest that misexpression of Lasp 1 is the most likely cause for the mutant defects. These data reveal a yet unappreciated role of Lasp1 in chondrocyte differentiation during cartilage to bone transition.

\section{Results}

\section{Phenotypic characterization of the insertional mutant Tg(Col1a1-lacZ)304ng (wavy tail)}

Only one of several transgenic lines created with the same DNA construct (see Methods) exhibited developmental abnormalities, and only when bred to homozygosity. (Heterozygous carriers remained asymptomatic). Even homozygous animals appeared entirely normal at birth and accordingly, no deviations from the normal phenotype were detectable in embryos or fetuses. The first signs of a skeletal defect became apparent at the end of the first postnatal week. Homozygous mutant animals had multiple bends of the tail but not kinks. This deformation was most pronounced at $\sim 15$ days after birth (Fig. 1). Transgenic mice were fertile and viable. The phenotype was observed in $100 \%$ of all mutant animals and was maintained upon breeding the mutation into a different mouse strain (C57BI/6).

Longitudinal histological sections of 10 day (P10) mutant tails showed vertebral bodies of normal thickness but reduced length (Fig. 1 A,B). At P10 growth plates appeared compressed and disrupted, mainly due to thinner zones of proliferating and hypertrophic cartilage; the wavy tai/phenotype combines a reduction in size and alterations in the shape of the tail vertebrae (Fig. $1 \mathrm{C}-\mathrm{F}$ ). As determined by BrdU incorporation, the number of proliferating
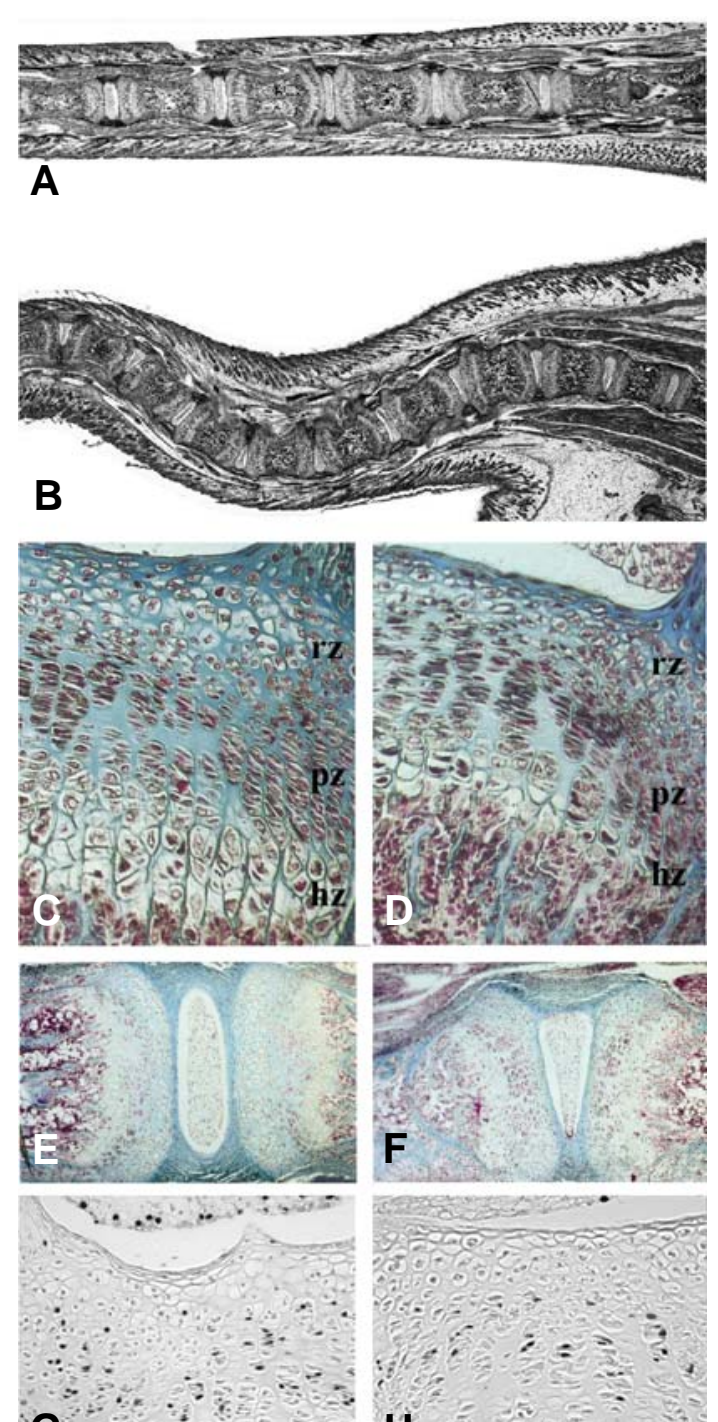

G
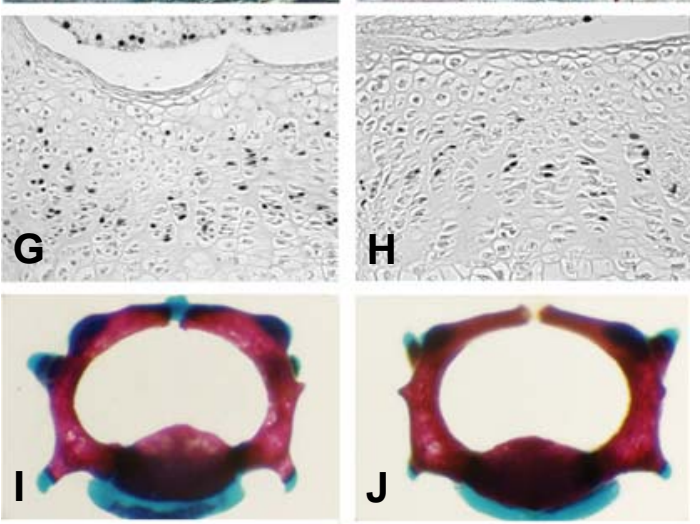

Fig. 1. Wavy tail Tg(Col1a1-lacT)304ng mutants have compressed tail vertebrae and wedge-shaped intervertebral discs. Longitudinal sections through wild type (A) and Tg(Col1a1-lacZ)304ng (B) tail of P10 animals showing scoliosis of the tail and decreased size of tail vertebrae. $P 10$ tail vertebrae of wild type (C) and mutant (D) P10 animals stained with Azan. Mutant intervertebral discs (F) are wedge-shaped and the proliferation zone of mutant vertebrae is compressed compared to wild type control samples (E). BrdU incorporation demonstrates a significant reduction in DNA synthesis of mutant chondrocytes. Quantification of BrdU positive cells in serial sections of the same level of P10 tail vertebrae revealed a significantly reduced number of proliferating cells in mutant $\mathbf{( H )}$ compared to wild type (G) tail vertebrae and intervertebral discs. Alizarin red alcian blue staining of the $3^{\text {rd }}$ lumbal vertebra of P10 wild type (I) and mutant animals $\mathbf{( J )}$; mutant neural arches failed to close in time and the processus spinosi were missing in the lumbal vertebral column at this stage. Abbreviations: hz, hypertrophic zone; pz, proliferating zone; $r z$, resting zone. 
cells was significantly reduced in mutant growth plates (Fig. $1 \mathrm{G}, \mathrm{H}$ ). Proliferating cells were determined by counting BrdU positive cells in longitudinal serial epiphyseal sections of three different wild type and mutant $\mathrm{P} 10$ tail vertebrae $\mathrm{n}(\mathrm{wt})=30$; mean value 44,8 $\pm 6,3 \mathrm{SD}$; $\mathrm{n}($ wavy tai $)=27$; mean value $32,7 \pm 11,5 \mathrm{SD}(\mathrm{df}=55$ two sample t-test $\mathrm{p}=0.00006)$.

The reduced proliferation resulted in the formation of considerably shorter (reduced to $2 / 3$ ) tail vertebrae compared to wild type controls, but vertebrae were still straight. (Fig. 1 E,F). The intervertebral discs were also shorter and, most conspicuously, were grossly deformed and wedge-shaped. In horizontal sections, only every third disc was found to be symmetric, whereas the others were strongly deformed with regular alteration of the orientation of the «wedge», producing the characteristic multiple smooth bends of the mutant tail without causing kinks. Quantification of BrdU positive cells in serial sections of the same level of P10 tail vertebrae demonstrated a significantly reduced number of proliferating cells in mutant intervertebral discs $n(w t)=15$; mean value $15,6 \pm 4,3 \mathrm{SD} ; \mathrm{n}$ ( wavy tai $)=12 ;$ mean value 10,6 $\pm 4,2 \mathrm{SD} p=0.006$, $\mathrm{df}=25$ (Fig. $1 \mathrm{G}, \mathrm{H})$. No defects in long bone and skull morphology were observed, thickness and length of these bones seemed unaffected in homozygous transgenic animals (data not shown).

Alcian blue and alizarin red skeletal staining demonstrated delayed closure of neural arches in the lumbal region. In the wild type, neural arches closed around P10, the most dorsal part and the processus spinosi still being cartilaginous; in contrast, the neural arches of mutant animals were still open and no processus spinosi were present at the same stage (Fig.1 I,J). Eventually, closure of the neural arches was completed in mutants at P14, the animals thus exhibiting only temporarily the symptoms of spina bifida occulta. In contrast to the full penetrance of tail scoliosis,

Fig. 2. Transgene arrangement and chromosomal location site. Chromosomal location of the transgene insertion site (A). The insertion site was mapped between the Col1a1 and the Wnt3 locus, it always cosegregated with Zfp144 on chromosome 11 band D (Kleiter et al., 2000). A screen for transcribed regions showed that six genes (Zfp144-Psmb3Pip5k2b-Mllt6-Rpl23-Lasp1) are located in a 200kb area surrounding the insertion site (Kleiter et al., 2002). LacZ staining of transgenic tail vertebrae (B). P10 mutant tail vertebrae show beta-galactosidase staining in bone and periosteum in blue. The distribution is similar to that observed for endogenous Col1a1 transcription. A schematic drawing illustrates location and arrangement of the transgene. The insertion is localized $6,785 \mathrm{~kb}$ upstream of the Lasp1 transcription start. A $3.5 \mathrm{~kb}$ fragment of the Col1a1 upstream regulatory region inserted next to Lasp1, this part of the upstream (-5.3 to -8.7$)$ regulatory region was oriented in the same transcriptional direction as the Lasp1 gene. The adjacent part of the first intron of Col1a1 is located in the same transcriptional orientation. A complete copy of the transgene consisting of the Col1a1 upstream region (-8.3 to $0 \mathrm{~kb}$ ) exon 1 and intron 1 fused to $\mathrm{nls} \mathrm{LaCz}$ was located in the opposite transcriptional direction. (C) VISTA Global Alignments of the mouse with rat and human Lasp1 loci. VISTA plot showing sequence similarity in pairwise sequence alignments between the mouse, rat and human sequences, respectively: Peaks are shown relative to their position in the reference mouse sequence (horizontal axis) and percent identity (50-100\%) is shown on the vertical axis. Color code is predominantly pink (non coding sequence), exons are illustrated in blue. Several conserved regions (mouse to rat) are located in the area of the transgene insertion, suggesting the presence of transcriptional control elements; the arrow indicates the transgene insertion site. The arrowhead denotes transcriptional orientation of the Lasp1 gene. delayed closure of the neural arches in the lumbar vertebrae was observed in roughly $50 \%$ of mutant animals. No alteration in the proliferation rate of chondrocytes in dorsal neural arches was observed by BrdU incorporation and staining of histological sections in P10 and P14 old animals (data not shown).

\section{Lasp1 expression is altered in wavy tail Tg(Col1a1-lacZ)304ng mutant mice}

The location of the transgene insertion site in close vicinity to Lasp1 and the presence of conserved genomic sequence in this

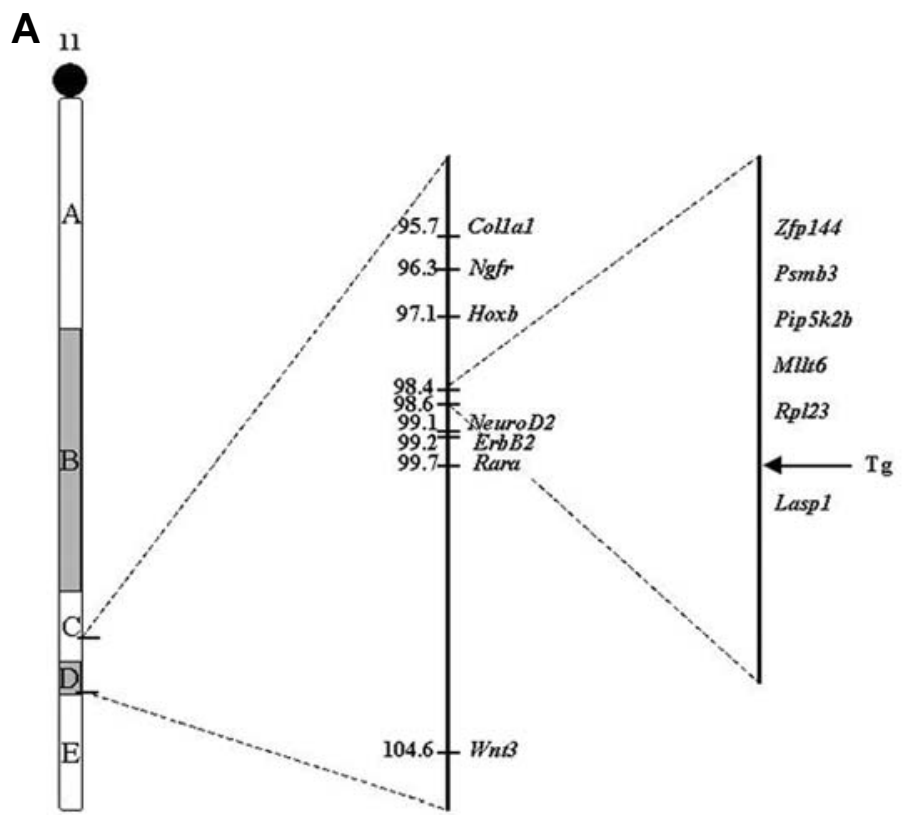

B

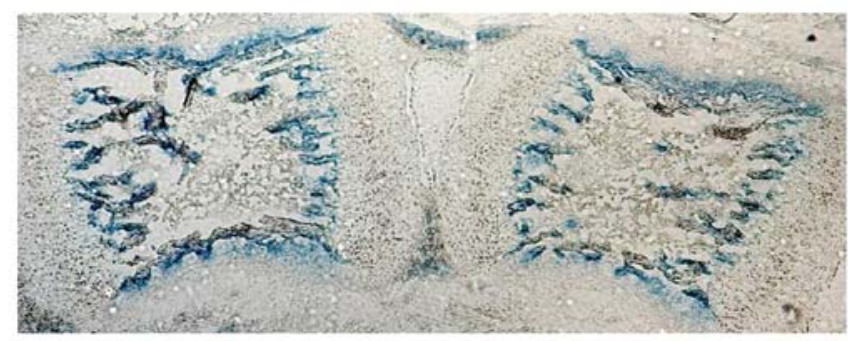

5 flanking region

transgene arrangement
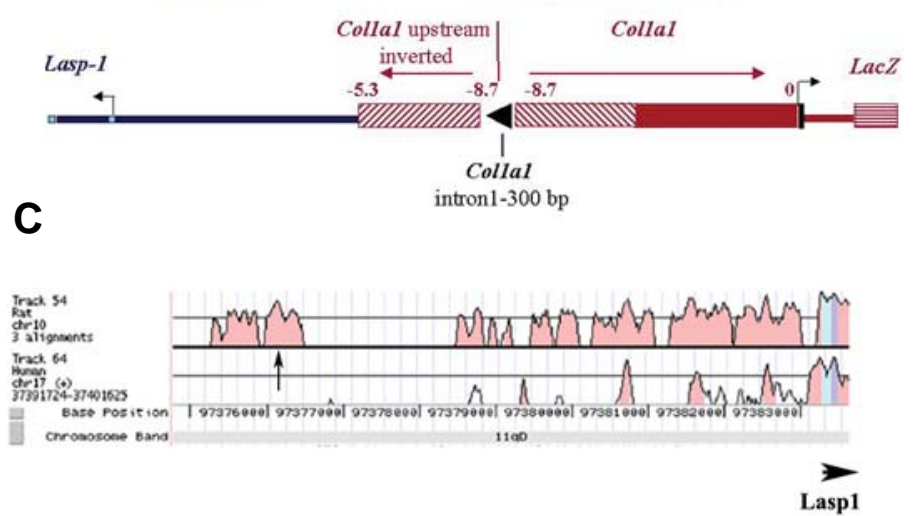


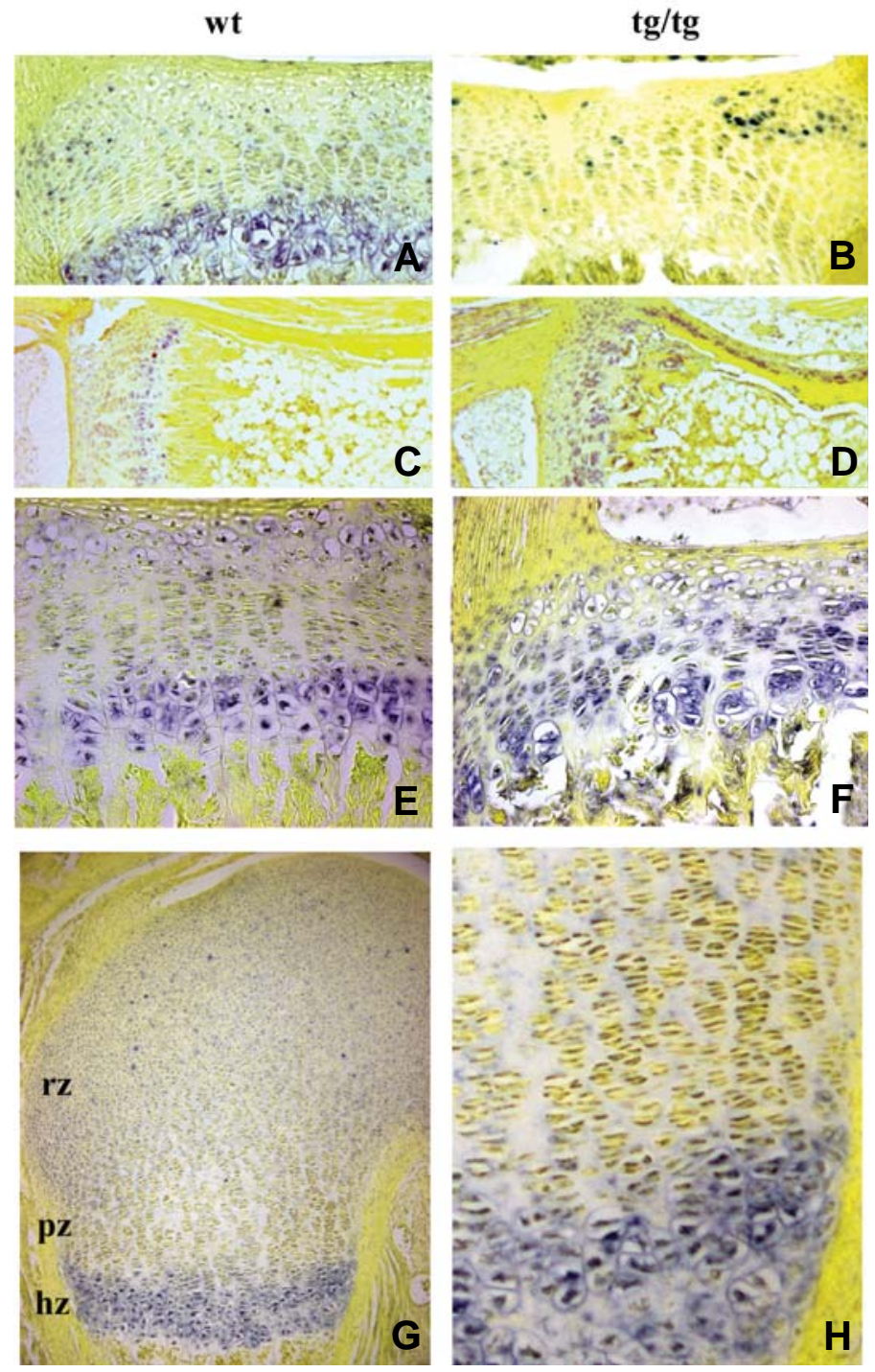

Fig. 3. Lasp1 expression in developing bones. Lasp1 expression in $P 7$ $(\mathbf{A}, \mathbf{B})$ and P14 (C-F) tail vertebrae and E18.5 long bones $(\mathbf{G}, \mathbf{H})$. Lasp1 is strongly transcribed in hypertrophic chondrocytes and to lesser extent in chondrocytes of the resting zone of wild type P7 (A) and P14 (E) growth plates, whereas the proliferative zone is devoid of Lasp1 expression. In P7 mutant growth plates (B) Lasp1 was detected in single cells of all zones, with the exception of the hypertrophic area. Ectopic expression of Lasp1 is detected in P14 mutant periosteum (D), whereas no Lasp1 mRNA can be found in wild type (C) periosteum. A detailed view of P14 growth plates shows that Lasp1 is expressed in hypertrophic and resting chondrocytes of wild type (E) tissue. In contrast all mutant chondrocytes misexpressed Lasp1, including the compressed proliferative zone (proliferative zone: Lasp1+ cells $n(w t)=9$; mean value 16.3 $13.6 S D ; n$ (wavy tail)=9; mean value $85.3 \pm 16 S D$ ( $d f=15$ two sample $t$-test $p=4.13 e-8)$. (F). Abbreviations: hz, hypertrophic zone; $p z$, proliferating zone; $r z$, resting zone.

area (Fig. 2) suggested Lasp1 as the most likely candidate gene affected in this insertional mutant. In addition, expression of the other genes close to the insertion site was not altered (Kleiter et al., 2002).

We examined the expression of $L a s p 1$ during skeleton forma- tion. Lasp1 transcripts were present in forming vertebrae as early as E14.5 and strong expression was detected in E17.5 hypertrophic chondrocytes (data not shown). Lasp 1 was also expressed in resting and hypertrophic chondrocytes in E18.5 femur (Fig. 3 $\mathrm{G}, \mathrm{H})$,

In P7 and P14 wild type tail vertebrae, Lasp1 mRNA was detected in hypertrophic and resting chondrocytes of the growth plate, while no expression was found in proliferative cells (Fig. 3 $A, B, E)$. Interestingly, we found an altered expression pattern in mutant vertebral growth plate tissue: at P7 no Lasp1 transcripts were detected in hypertrophic chondrocytes, and only sporadic expression was observed in individual cells throughout all zones of the growth plate (Fig. 3B). Then at P14, Lasp1 was transcribed in cells of all stages of chondrocyte differentiation (resting, proliferative and hypertrophic) in mutant tail vertebrae (Fig. $3 \mathrm{D}, \mathrm{F}$ ). In addition, ectopic expression was observed in the periosteum of tail vertebrae (Fig. 3D).

A characteristic feature of the wavy tailmutant is a temporary spina bifida occulta in the lumbar region. Lasp1 expression was therefore examined in lumbar vertebrae at P8. Transcripts were found in resting and hypertrophic chondrocytes in a pattern that was not obviously different between wild type and mutant vertebrae (data not shown). In addition, ectopic expression was found in the periosteum of the mutant.

\section{Mutant animals are sensitive to low doses of retinoic acid and are folate resistant}

Retinoic acid (RA) is known to strongly affect the development of the vertebral column (Kessel and Gruss, 1991; Lohnes et al., 1993). Moreover, administration of low doses of retinoic acid can reduce the incidence of neural tube defects (Chen et al., 1994; Seller and Perkins, 1982). Remarkably, wavy tail mutant animals exhibited increased sensitivity to RA. Low doses (10 mg/kg body weight) applied at day 9 of gestation caused perinatal death in all (17) animals born, but not in control animals which appeared asymptomatic (11). In contrast to the wild type situation, ossification centers of the cervical vertebrae were absent in $50 \%$ of the transgenic mice treated (Fig. 4F). In addition, the $6^{\text {th }}$ lumbal vertebra was missing in 8 mutant animals (Fig. 4D). Furthermore malformations in the sternum were detected, as all RA treated neonates exhibited a widened and split xyphoid process, and the formation of a "crankshaft sternum» (asymmetrically attached ribs) was observed occasionally (Fig. 4 $A, B$ ). Unfortunately, due to the early death of the animals (defects in breathing caused by formation of a cleft palate), effects of RA on neural arch development in the lumbar region could not be further studied in RA-treated mice.

Clinical trails in humans have shown that maternal folic acid supplements prior to and during early pregnancy can prevent both the occurrence and the recurrence of neural tube defects (NTD). Nevertheless the mechanism by which folic acid prevents NTD is still poorly understood. We investigated the effect of folic acid administration in the wavy tai/mutant. The $50 \%$ incidence of spina bifida was not changed in treated animals (data not shown) indicating that our mutant is folate-resistant.

\section{Aberrant expression of collagen genes and Pax1 in wavy tail $\mathrm{Tg}$ (Col1a1-lacZ)304ng mutant animals}

To further characterize the defective development of mutant vertebral growth plate, we analyzed the expression of skeletal 
marker genes. Collagens are components of the extra cellular matrix (ECM) of cartilage and bone. Type II collagen is the major and the characteristic collagen of cartilage and is expressed in all chondrocytes. In situ hybridization (ISH) for Col2a1 performed on P14 tail vertebrae showed that in the wild type, all chondrocytes of the growth plate expressed CoRa1 (Fig. 5A). In the resting zone of mutant animals, sporadic cells failed to express Col2a1, while in the proliferative zone, whole chondrons were devoid of Col2a1 transcripts ( $30 \%$ of proliferating cells lacked Col2a1 transcripts, Fig. 5B). In most cases all cells of an individual chondron failed to express Col2a1, suggesting that these cells had arisen from a single non-expressing cell in the resting zone.

Collagen type $\mathrm{X}$ is a marker for terminally differentiating chondrocytes of the prehypertrophic and hypertrophic zones in the growth plate (Kong et al., 1993). Prehypertrophic chondrocytes produce collagen $\mathrm{X}$, but do not secrete the protein. Once the cells become hypertrophic, type $X$ collagen is deposited into the ECM. By immunohistochemistry we found that collagen type $X$ is produced both in wild type and mutant tail vertebrae (Fig. 5 C,D); mutant hypertrophic chondrocytes, however, failed to secrete the
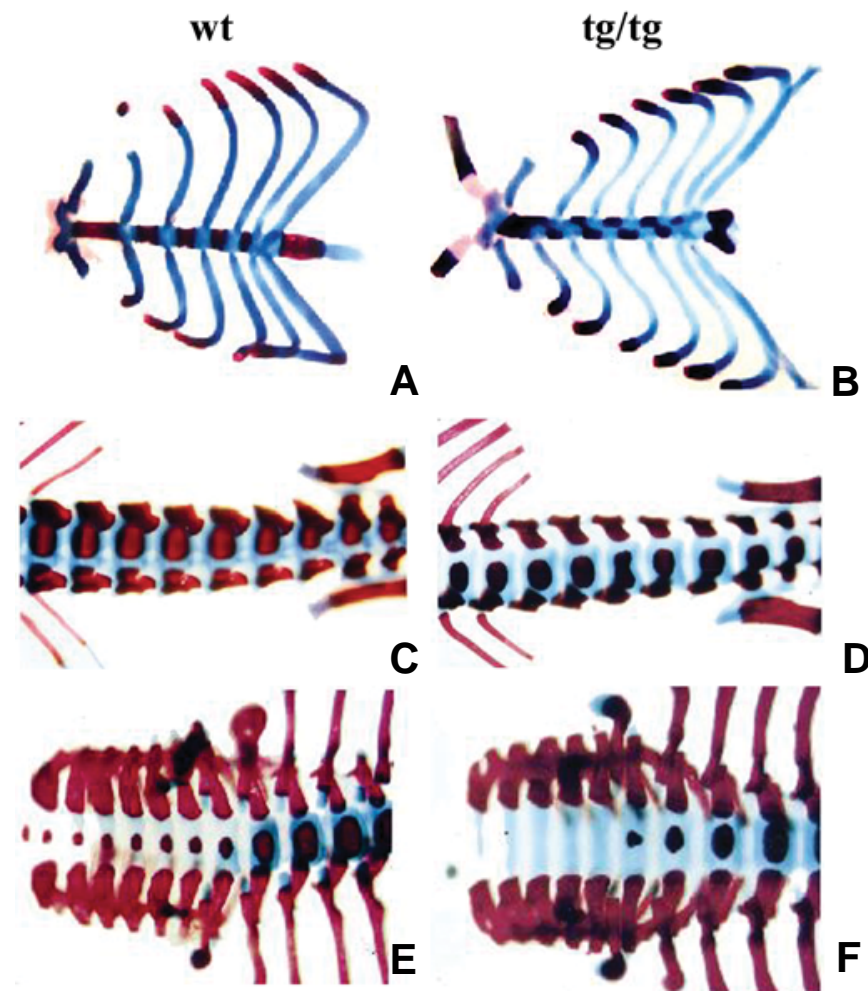

protein (Fig. 5F), suggesting a defect in chondrocyte terminal differentiation.

Collagen type I is the major constituent of bone ECM while it is absent in (most) cartilage. It is therefore a marker of beginning osteogenesis. P14 wildtype and mutant vertebrae were analyzed for transcripts of Colla1, which is expressed in osteoblasts, the perichondrium and the periosteum. In some mutant vertebrae expression of Col/a1was specifically reduced in osteoblasts (Fig. $5 \mathrm{H}$ ), whereas its transcription appeared to be unaffected in the perichondrium and the periosteum. Expression of the non-collagen chondrocyte markers Ihh, PTHrP and Bmp6 showed no differences between wild type and mutant tail vertebrae (data not shown).

The murine paired box-containing gene Pax1 is required for normal development of the vertebral column, the sternum, and the scapula (Balling et al., 1992). Expression of Pax1 was examined

wt

Col $2 a 1$

ISH
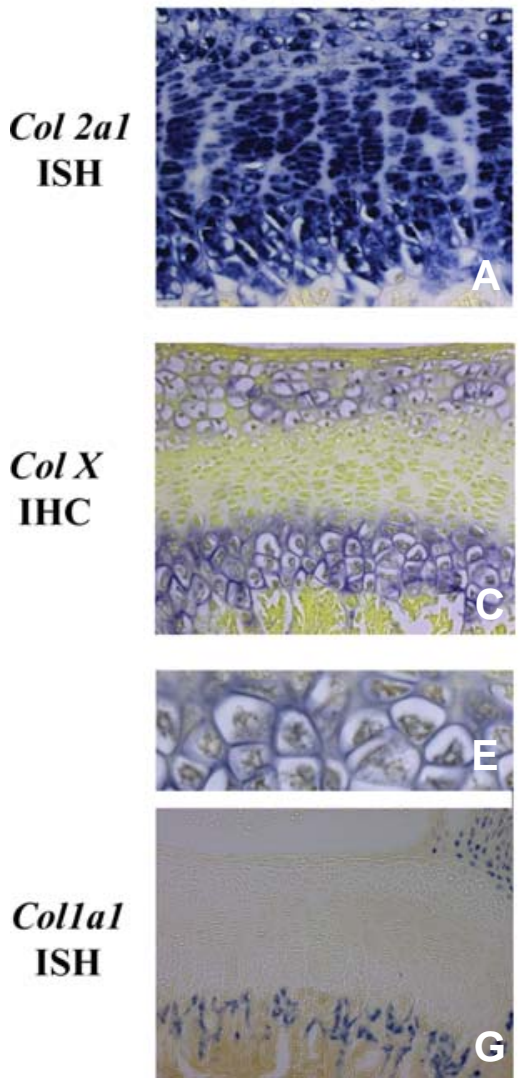

$\operatorname{tg} / \operatorname{tg}$
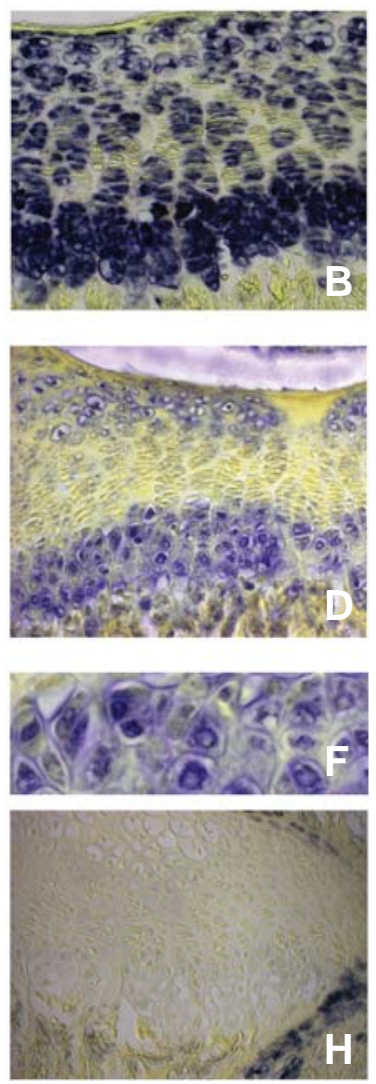

Fig. 4 (Left). Effect of retinoic acid (RA) on skeletal development of wild type and Tg(Col1a1-lacZ)304ng mutant neonates. Analysis of skeletal elements of wild type and Tg(Col1a1-lacZ)304ng mutant neonates treated with $10 \mathrm{mg} / \mathrm{kg}$ RA at E9.5. Whole mount skeletons were prepared from wild type $(A, C, E)$ and mutant $(B, D, F)$ neonates. Ventral view of the sternum shows symmetric fusion of the sternbrae ossification centers in wild type (A) sternum. In contrast, mutant neonates (B) exhibit shifted fusion of the ossification centers resulting in a crankshaft sternum. Dorsal view of the lumbal vertebral column: 6 lumbal vertebrae in wild type (C) but only 5 vertebrae in mutant (D) animals. Dorsal view of the cervical vertebrae ossification centers in the wild type (E), these centers are missing in mutant neonates $\mathbf{( F )}$

Fig. 5 (Right). Chondrocyte marker gene expression in P 14 wild type and Tg(Col1a1-lacZ)304ng mutant tail vertebrae. Analysis of chondrocyte marker gene expression using in situ hybridization and immunohistochemistry on P14 wild type and Tg(Col1a1-lacZ)304ng mutant tail vertebral sections. Wild type chondrocytes (A) express Col2a1 throughout the growth plate. In mutant growth plates (B) Col2a1 expression is lost in single cells and chondrons respectively. COLX protein is expressed and secreted in wild type hypertrophic chondrocytes (C, E). Mutant hypertrophic chondrocytes produce COLX (D), but fail to secrete it (F). Col1a1 transcripts are detected in wild type osteoblasts, periosteum, and perichondrium (G), mutant samples exhibited a loss of expression selectively in osteoblasts $\mathbf{( H ) , ~ w h e r e a s ~ t r a n s c r i p t i o n ~ i n ~ t h e ~ p e r i c h o n d r i u m ~ s e e m s ~ t o ~ b e ~ u n a f f e c t e d . ~}$ 

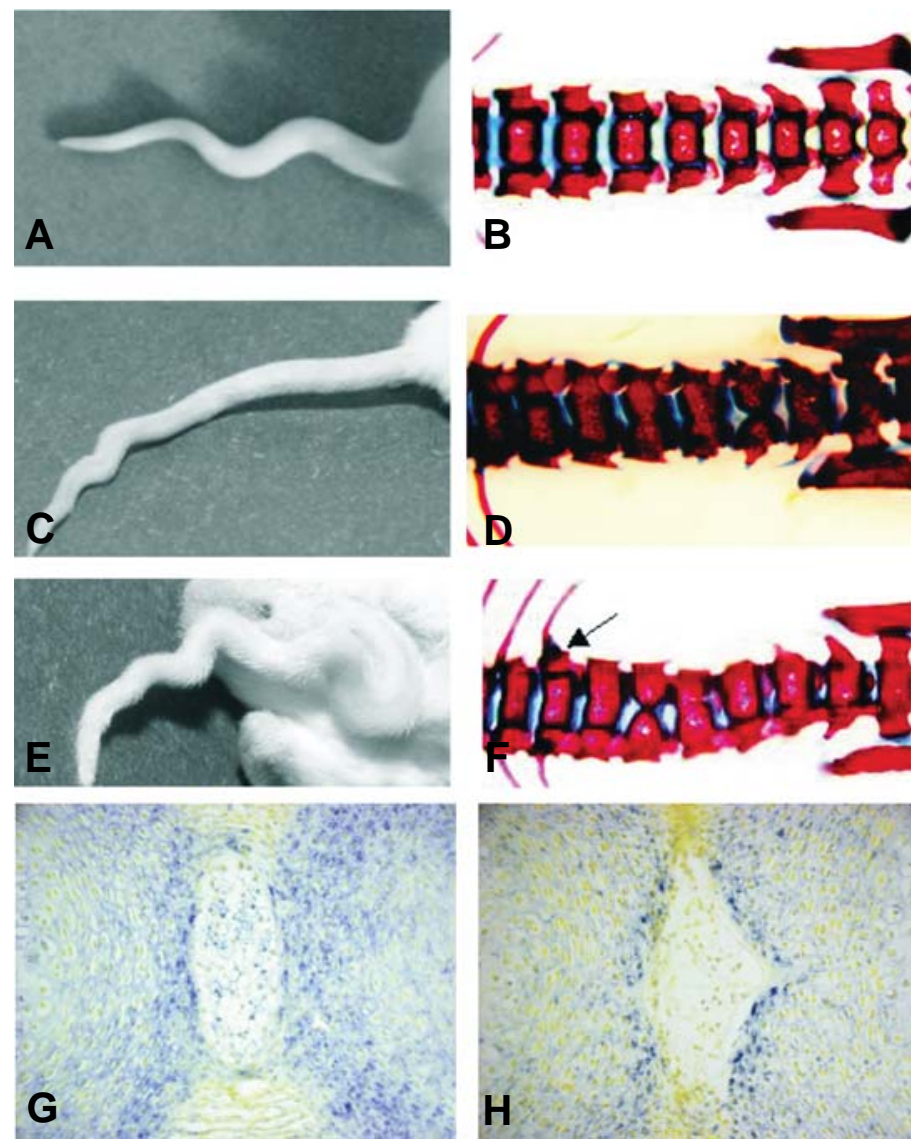

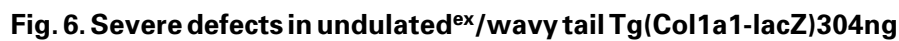
double mutants. Skeletal structures of Tg(Col1a1-lacZ)304ng/Tg(Col1a1lacZ)304ng, unex/unex, and unex/unex/ Tg(Col1a1-lacZ)304ng/Tg(Col1a1lacZ)304ng compound mutants. The tail vertebral column of wavy tail mutants (A) presents with scoliosis, the tail is bent but, in contrast to the $u^{e x} / n^{e x}$ mutant (C), has no kinks. Compound mutant tails (E) develop kinks, bends and a strong upbending of the vertebral column immediately after the sacral region. Ventral view of the lumbal vertebral column showing a structure similar to that of wild type vertebral bodies in the

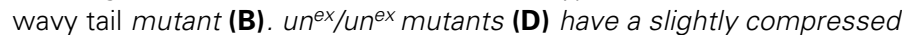
vertebral column. Double mutant animals (F) show stronger compression of the vertebrae, split vertebral bodies and a thickened ribjoint. Analysis of $\mathrm{Pax} 1$ expression using in situ hybridization. E18 wild type tail vertebrae (G) expressed Pax1 in the condensed cartilage of the vertebrae, the intervertebral discs, and the tissue surrounding the intervertebral discs. In mutant vertebrae (H) expression was weaker and completely missing in the intervertebral discs.

by in situhybridization. At E10.5 whole-mount in situhybridization revealed no differences between wildtype and mutant embryos (data not shown). However, at E18.5, when Pax1 expression was found in vertebral chondrocytes and in intervertebral discs of wild type samples (Fig. 6G), no Pax1 transcripts were detected in intervertebral discs of wavy tail mutant animals (Fig. $6 \mathrm{H}$ ), suggesting either a direct interaction with the Pax1 gene, or absence/ delayed maturation of intervertebral discs.

\section{Severe defects in undulated ${ }^{e x} /$ wavy tail $\mathrm{Tg}$ (Col1a1-lacZ)304ng double mutant animals}

Previous studies have shown that three natural Pax1 (undu- lated) mouse mutants displayed phenotypes of different severity in axial skeletal elements (Balling etal., 1992). WavytailTg(Col1a1lacZ)304ng mutants showed similar defects. To explore a possible genetic interaction, double mutant mice with undulatedextensive (un ${ }^{\mathrm{ex}}$ ) were generated. un ${ }^{\mathrm{ex}}$ homozygous animals have skeletal defects in the lumbal region and the tail ((Dietrich and Gruss, 1995; Wallin et al., 1994); Fig. 6 C,D). At birth, double mutant animals showed a similar phenotype to $u n^{\mathrm{ex}} / u n^{\mathrm{ex}}$. However, during the first week of postnatal development, i.e. when the phenotype of our mutant first becomes apparent, tails of double mutant mice sharply bent upwards immediately after the last sacral vertebra, forming a hair pin turn-like structure (Fig. 6E). Deformation of the tail was stronger than in each single mutant (Fig. 6 E,F). In addition, compressed lumbar vertebrae with split vertebral bodies (ventral fusion failed to occur), as well as thickened rib joints were seen (Fig. 6 compare wavy tail(B), un $n^{e x} u n^{e x}$ (D) to double mutant (F)). Tg/Tg; $u n^{e x /}+$ and $\mathrm{Tg} /+; u n^{e x} u n^{e x}$ animals showed no differences to either single homozygous mutant.

\section{Discussion}

The most remarkable feature of wavy tail insertional mutant animals is that the mutant phenotype becomes apparent only after birth. We were unable to detect abnormalities in embryos and fetuses, and even at birth, homozygous animals were completely asymptomatic. Curvature of the tail started to develop only during the second postnatal week, coinciding with the juvenile growth phase (the phenotype becoming most pronounced at P14). These features suggested that the dysmorphogenesis was due to neuromuscular disorders. In humans the most common forms of scoliosis are associated with spinal muscular atrophy which is a neuromuscular spinal deformity with a long c-shaped curve. Single curves are seen in approximately $90 \%$ of patients. Thoracolumbar curves are most common, occurring in $80 \%$, whereas thoracic curves are seen in approximately $20 \%$ of patients (Sucato, 2007). However, the" wavy tail mouse mutant did not develop any of these characteristics, as only the tail was deformed and only during postnatal development. Other mouse models developed defects in the lumbal region or tail already around midgestation, like in the wavy tai/mutant these malformation were restricted to the vertebral column but not muscular development (flaky tail (Rothnagel et al., 1994), TgN(Imunsd)379Rpw (Schrick et al., 1995), Sickle tail (Semba et al., 2006), Jun (Behrens et al., 2003), Delta- like I(Teppner et al., 2007), or Tgfbr2 (Baffi et al., 2006)).

Analysis of the genomic sequence surrounding the insertion site showed that no open reading frame was disrupted, however the transgene inserted in a conserved region close to the Lasp1 transcription start site (Fig. 2A). This suggests Lasp1 as a likely candidate gene affected in the mutant. Interestingly, we found that $L a s p 1$ is transcribed in the wild type growth plate, specifically in resting and hypertrophic chondrocytes. In contrast, Lasp1 expression is altered in mutant tail vertebrae (it is ectopically expressed in all zones of the growth plate and misexpressed in the periosteum) which further indicates that the transgene insertion influences $L a s p 1$ transcription.

The transgene inserted approx. $6.7 \mathrm{~kb}$ upstream of the $\angle a s p 1$ transcription start. Vista plot analysis demonstrated that this area 
is conserved between rat and mouse (Fig. 2C), suggesting that the insertion disrupted an upstream regulatory element of the Lasp1 gene, which could result in misexpression/lack of expression in chondrocytes. On the other side, the transgene itself contains strong Col1a1enhancer sequences, which could lead to ectopic expression of Lasp1 in the Col1a1 expression domain. The periosteum expresses high levels of Col1a1, thus the ectopic expression of Lasp1 in this tissue may be caused by the close vicinity of the Col1a1 enhancer to the Lasp1 transcription start.

Experiments in NIH3T3 cells indicated that LASP1 is required for cell migration and survival (Lin et al., 2004), the mis- or overexpression of Lasp1 could lead to changes in chondrocyte cell migration, survival, and differentiation especially during periods of extensive growth (e.g. in the postnatal growth phase). Both increase and depletion of Lasp1 expression in COS-7, HEK293 and MCF-7 cells inhibited basal and growth factor-stimulated cell migration (Lin et al., 2004) and knock-down of Lasp1 in metastatic breast cancer lines resulted in inhibition of proliferation and migration due to a reduction of zyxin at focal contacts (Grunewald et al., 2006). LASP1 is also associated with actin (Butt etal., 2003; Schreiber et al., 1998b), therefore lack of expression or misexpression could lead to structural changes of the chondrocyte cytoskeleton, resulting in a disorganized appearance of growth plates. This becomes even more apparent when examining Col2a 1 expression, which is partially lost in mutant chondrocytes. In contrast, Col $X$ was still expressed in hypertrophic chondrocytes, but secretion (of the protein) was inhibited, which further argues for defects in chondrocyte differentiation in the mutant. Interestingly, Pax1 expression was unaltered early during development (before manifestation of the wavy tai/phenotype), but was lost in wavy tail mutant intervertebral discs, suggesting an absence of, or delayed maturation of these chondrocytes. Pax 1 is involved in transducing proliferative signals from the notochord to sclerotome cells during skeleton formation (Furumoto et al., 1999). Thus the lack of Pax 1 expression could be responsible for the reduced proliferation rate found in mutant chondrocytes.

Neural tube defects that can be prevented by folic acid provide excellent model systems to investigate its protective mechanism. Administration of folic acid results in a significant improvement of neural tube defects in some mutant mice strains like Cart1 (Zhao et al., 1993); Cited2 (Barbera et al., 2002) and splotch (Epstein et al., 1991). On the other side, folate-resistant mutants offer the possibility to detect new pathways for NTD prevention, as has been demonstrated in the curly tail mutant, where inositol prevents NTD (Greene and Copp, 1997). The wavy tail mutant is folate-resistant, as the spina bifida occulta was not responsive to the administration of folic acid. In previous studies, folate-resistant neural tube defects were improved by alterations of RA signaling (Corcoran, 1998). In strong contrast, wavy tai/Tg(Col1a1lacZ)304ng mutant embryos develop even more severe skeletal defects upon RA administration at E9.5. We could not identify RA response elements in the $L a s p 1$ regulatory sequences suggesting that RA signaling does not directly regulate Lasp1expression.

We have characterized a novel transgene insertional mutant with distinct skeletal defects in vertebral column formation. This phenotype was linked to deregulation of Lasp1 gene transcription, which had not been associated with chondrocyte differentiation and function before. The known association of Lasp 1 with the cytoskeleton suggests that mutant animals experience defects in cytoskeletal organization, which are most pronounced during the postnatal growth phase of vertebral bones. Lasp1over-expression seems to have a negative effect on cartilage cell proliferation, on Collagen type X, Col2a1, Col1a1 and Pax1, but not on the expression of the non-collagen chondrocyte markers Ihh, PTHrP and $B m p 6$. In order to investigate these effects and to validate the important role of Lasp1 during cartilage to bone transition, analysis of deletion mutants or transgenic over-expression models will be required.

\section{Materials \& Methods}

\section{Mice}

Transgenic animals have been produced by pronuclear injection of a linearized $15 \mathrm{~kb}$ DNA fragment containing Col1a1 and n/s-LacZ sequences. The reporter gene construct injected contained the transcription start site of the murine Col1a1gene with $8.7 \mathrm{~kb}$ of upstream sequences, the entire exon1 (2 ATG codons mutated to TTG), intron1 (including 47bp of the LTR of the Moloney murine leukemia virus in the Mov13 insertion site (Harbers et al., 1984), and 13bp of exon2. These sequences were fused to the bacterial $\angle a c Z$ reporter gene (including the small intron and poly[A] signal of SV40 T) to yield a construct of $15.0 \mathrm{~kb}$ (Fig. 2B). As reported before (Kleiter et al., 2002), a few (3-5) copies of the construct have inserted as concatemers with minor rearrangement within the insert (Fig. 2B). X-gal staining revealed that expression of this reporter gene faithfully reflected transcription of the endogenous Col1a1gene (Fig. 2B, unpublished results, Ghaffari-Tabrizi N.). The transgenic mouse line was originally produced in NMRI mice, and was bred to homozygosity in both FVB and C57BI/6 mouse strains. Heterozygosity was determined by Southern blot analysis using a lacZ reporter gene probe or by lacZstaining of P7 tail tips (Kleiter et al., 2000). As dizygous carriers of the insert were viable and fertile, the line was bred in homozygous condition. Dated pregnancies were obtained by detection of a vaginal plug (=E0.5). The mouse strain undulatedcontaining the Pax1 mutation un ${ }^{\mathrm{ex}}$ was a gift from Rudi Balling (GBF Braunschweig).

\section{Skeletal analysis}

Animals were sacrificed, skinned, and eviscerated. Alcian blue and Alizarin red whole mount skeletal staining was performed as previously described (Kessel and Gruss, 1991).

\section{In situ hybridization and immunohistochemistry}

Tissues were fixed overnight in $4 \%$ freshly prepared paraformaldehyde in PBS, skeletal components were decalcified in $2.5 \%$ paraformaldehyde/ $12.5 \%$ EDTA in PBS for 1 week. Samples were subsequently embedded in paraffin. In situ hybridization was performed on $8 \mu \mathrm{m}$ section according to (Henrique et al., 1995). Col1a1, Col2a1, and Pax1 in situ probes were generated as previously described (Aigner et al., 1995; Jaenisch et al., 1983; Peters et al., 1995). Plasmids containing Lasp1 (clone ID IMAGp998F101146) cDNA clones were obtained from the resource center of the human genome project (www.rzpd.de).

Immunohistochemistry was performed on $8 \mu \mathrm{m}$ sections. Sections were dewaxed and rehydrated. Proteinase $\mathrm{K}$ digestion was used for antigen retrieval prior to overnight incubation with the primary antibody solution (rabbit anti-ColX, 1:1000, BR Olsen, Harvard Medical School). Secondary antibody was goat anti-rabbit alkaline phosphatase-conjugated (Biorad), and color development was performed using BM Purple AP substrate (Roche).

\section{LacZ staining}

Beta galactosidase detection on cryo-sections was described previously (Schwarzler et al., 1997).

\section{Administration of retinoic acid}

All-trans retinoic acid was dissolved in corn oil and injected intraperi- 
toneally into pregnant females (E9.5) at $10 \mu \mathrm{g} / \mathrm{g}$ body weight. Newborn animals were sacrificed and stained with Alcian blue and Alizarin red (Kessel and Gruss, 1991).

\section{Folic acid treatment}

Prenatal folic acid treatment began the day of vaginal plug discovery. Folic acid ( $3 \mathrm{mg} / \mathrm{kg}$ body weight; Sigma) was administered daily by intraperitoneal injection. PBS was used as the vehicle for these injections. Animals were assessed morphologically for the presence or absence of NTDs.

\section{BrdU incorporation}

Animals were injected intraperitoneally with bromo-deoxyuridine (BrdU) solution $(50 \mathrm{mg} / \mathrm{kg}$ body weight in $0.1 \mathrm{M}$ Tris- $\mathrm{HCl}$ buffer (Sigma)) and sacrificed two hours later. Proliferating cells were stained with peroxidase-coupled anti-BrdU antibody (Chemicon) and hematoxylin eosin. A total of 56 growth zone and 26 intervertebral disc sections were analyzed for BrdU positive cells in three individual wild type and transgenic animals. Statistical analysis was performed using a two sample t-test.

\section{Acknowledgments}

We thank Klaus Kratochwil for scientific advice. This work supported by the Austrian Science Fund Project number S07402 MOB.

\section{References}

AIGNER, T., DIETZ, U., STOSS, H. and VON DER MARK, K. (1995). Differential expression of collagen types I, II, III, and X in human osteophytes. Lab Invest 73: $236-243$.

BAFFI, M.O., MORAN, M.A. and SERRA, R. (2006). Tgfbr2 regulates the maintenance of boundaries in the axial skeleton. Dev Bio/296: 363-374.

BALLING, R., LAU, C.F., DIETRICH, S., WALLIN, J. and GRUSS, P. (1992). Development of the skeletal system. Ciba Found Symp 165: 132-40; discussion 140-143.

BARBERA, J.P., RODRIGUEZ, T.A., GREENE, N.D., WENINGER, W.J., SIMEONE, A., COPP, A.J., BEDDINGTON, R.S. and DUNWOODIE, S. (2002). Folic acid prevents exencephaly in Cited2 deficient mice. Hum Mol Genet 11: 283-293.

BEHRENS, A., HAIGH, J., MECHTA-GRIGORIOU, F., NAGY, A., YANIV, M. and WAGNER, E.F. (2003). Impaired intervertebral disc formation in the absence of Jun. Development 130: 103-109.

BUTT, E., GAMBARYAN, S., GOTTFERT, N., GALLER, A., MARCUS, K. and MEYER, H.E. (2003). Actin binding of human LIM and SH3 protein is regulated by cGMP-and cAMP-dependent protein kinase phosphorylation on serine 146 . $J$ Biol Chem 278: 15601-15607.

CHEN, W.H., MORRISS-KAY, G.M. and COPP, A.J. (1994). Prevention of spinal neural tube defects in the curly tail mouse mutant by a specific effect of retinoic acid. Dev Dyn 199: 93-102.

CHEW, C.S., PARENTE, J.A., JR., ZHOU, C., BARANCO, E. and CHEN, X. (1998). Lasp-1 is a regulated phosphoprotein within the cAMP signaling pathway in the gastric parietal cell. Am J Physio/275: C56-C67.

CORCORAN, J. (1998). What are the molecular mechanisms of neural tube defects? Bioessays 20: 6-8.

DIETRICH, S. and GRUSS, P. (1995). undulated phenotypes suggest a role of Pax1 for the development of vertebral and extravertebral structures. Dev Bio/167: 529-548.

EPSTEIN, D.J., VEKEMANS, M. and GROS, P. (1991). Splotch (Sp2H), a mutation affecting development of the mouse neural tube, shows a deletion within the paired homeodomain of Pax-3. Cel/67: 767-774

FURUMOTO, T.A., MIURA, N., AKASAKA, T., MIZUTANI-KOSEKI, Y., SUDO, H., FUKUDA, K., MAEKAWA, M., YUASA, S., FU, Y., MORIYA, H. et al. (1999). Notochord-dependent expression of MFH1 and PAX1 cooperates to maintain the proliferation of sclerotome cells during the vertebral column development. Dev Bio/210: 15-29.

GREENE, N.D. and COPP, A.J. (1997). Inositol prevents folate-resistant neural tube defects in the mouse. Nat Med 3: 60-66.
GRUNEWALD, T.G., KAMMERER, U., SCHULZE, E., SCHINDLER, D., HONIG A., ZIMMER, M. and BUTT, E. (2006). Silencing of LASP-1 influences zyxin localization, inhibits proliferation and reduces migration in breast cancer cells. Exp Cell Res 312: 974-982.

HARBERS, K., KUEHN, M., DELIUS, H. and JAENISCH, R. (1984). Insertion of retrovirus into the first intron of alpha $1(\mathrm{I})$ collagen gene to embryonic lethal mutation in mice. Proc Nat/ Acad Sci USA 81: 1504-1508.

HELWIG, U., IMAI, K., SCHMAHL, W., THOMAS, B.E., VARNUM, D.S., NADEAU J.H. and BALLING, R. (1995). Interaction between undulated and Patch leads to an extreme form of spina bifida in double-mutant mice. Nat Genet 11: 60-63.

HENRIQUE, D., ADAM, J., MYAT, A., CHITNIS, A., LEWIS, J. and ISH-HOROWICZ, D. (1995). Expression of a Delta homologue in prospective neurons in the chick. Nature 375: 787-790.

JAENISCH, R., HARBERS, K., SCHNIEKE, A., LOHLER, J., CHUMAKOV, I., JAHNER, D., GROTKOPP, D. and HOFFMANN, E. (1983). Germline integration of moloney murine leukemia virus at the Mov13 locus leads to recessive lethal mutation and early embryonic death. Ce//32: 209-216.

KESSEL, M. and GRUSS, P. (1991). Homeotic transformations of murine vertebrae and concomitant alteration of Hox codes induced by retinoic acid. Ce//67: 89104

KIBAR, Z., CAPRA, V. and GROS, P. (2007). Toward understanding the genetic basis of neural tube defects. Clin Genet 71: 295-310.

KLEITER, N., ARTNER, I., COPELAND, N.G., GILBERT, D.J., JENKINS, N.A. and KRATOCHWIL, K. (2000). Genomic organization and chromosome location of the murine Rpl23 gene. Cytogenet Cell Genet 90: 227-230.

KLEITER, N., ARTNER, I., GMACHL, N., GHAFFARI-TABRIZI, N. and KRATOCHWIL, K. (2002). Mutagenic transgene insertion into a region of high gene density and multiple linkage disruptions on mouse chromosome 11 Cytogenet Genome Res 97: 100-105.

KONG, R.Y., KWAN, K.M., LAU, E.T., THOMAS, J.T., BOOT-HANDFORD, R.P., GRANT, M.E. and CHEAH, K.S. (1993). Intron-exon structure, alternative use of promoter and expression of the mouse collagen $X$ gene, Col10a-1. Eur $J$ Biochem 213: 99-111.

LIN, Y.H., PARK, Z.Y., LIN, D., BRAHMBHATT, A.A., RIO, M.C., YATES, J.R., 3RD and KLEMKE, R.L. (2004). Regulation of cell migration and survival by focal adhesion targeting of Lasp-1. J Cel/ Biol165: 421-432.

LOHNES, D., KASTNER, P., DIERICH, A., MARK, M., LEMEUR, M. and CHAMBON, P. (1993). Function of retinoic acid receptor gamma in the mouse. Cel/73: 643658.

NAKAGAWA, H., TERASAKI, A.G., SUZUKI, H., OHASHI, K. and MIYAMOTO, S. (2006). Short-term retention of actin filament binding proteins on lamellipodial actin bundles. FEBS Lett 580: 3223-3228

PETERS, H., DOLL, U. and NIESSING, J. (1995). Differential expression of the chicken Pax-1 and Pax-9 gene: in situhybridization and immunohistochemical analysis. Dev Dyn 203: 1-16.

ROTHNAGEL, J.A., LONGLEY, M.A., BUNDMAN, D.S., NAYLOR, S.L., LALLEY, P.A., JENKINS, N.A., GILBERT, D.J., COPELAND, N.G. and ROOP, D.R. (1994). Characterization of the mouse loricrin gene: linkage with profilaggrin and the flaky tail and soft coat mutant loci on chromosome 3. Genomics 23: 450456.

SCHREIBER, V., MASSON, R., LINARES, J.L., MATTEI, M.G., TOMASETTO, C. and RIO, M.C. (1998a). Chromosomal assignment and expression pattern of the murine Lasp-1 gene. Gene 207: 171-175.

SCHREIBER, V., MOOG-LUTZ, C., REGNIER, C.H., CHENARD, M.P., BOEUF H., VONESCH, J.L., TOMASETTO, C. and RIO, M.C. (1998b). Lasp-1, a novel type of actin-binding protein accumulating in cell membrane extensions. Mol Med 4: 675-687.

SCHRICK, J.J., DICKINSON, M.E., HOGAN, B.L., SELBY, P.B. and WOYCHIK, R.P. (1995). Molecular and phenotypic characterization of a new mouse insertional mutation that causes a defect in the distal vertebrae of the spine. Genetics 140: 1061-1067.

SCHWARZLER, C., WEBERSINKE, G., FASSLER, R., LAMETSCHWANDTNER A., BAUER, H. and BAUER, H.C. (1997). Incorporation of beta-galactosidaseexpressing endothelial cells into the skeletal muscle microvascular bed of mice. Cell Transplant 6: 9-15.

SEARLE, A.G., PETERS, J., LYON, M.F., HALL, J.G., EVANS, E.P., EDWARDS 
J.H. and BUCKLE, V.J. (1989). Chromosome maps of man and mouse. IV. Ann Hum Genet 53: 89-140.

SELLER, M.J. and PERKINS, K.J. (1982). Prevention of neural tube defects in curly-tail mice by maternal administration of vitamin A. Prenat Diagn2: 297-300.

SEMBA, K., ARAKI, K., LI, Z., MATSUMOTO, K., SUZUKI, M., NAKAGATA, N., TAKAGI, K., TAKEYA, M., YOSHINOBU, K., ARAKI, M. et al. (2006). A novel murine gene, Sickle tail, linked to the Danforth's short tail locus, is required for normal development of the intervertebral disc. Genetics 172: 445-456

SUCATO, D.J. (2007). Spine deformity in spinal muscular atrophy. J Bone Joint Surg Am 89 Suppl 1: 148-154.

TEPPNER, I., BECKER, S., DE ANGELIS, M.H., GOSSLER, A. and BECKERS, J (2007). Compartmentalised expression of Delta-like 1 in epithelial somites is required for the formation of intervertebral joints. BMC Dev Bio/7: 68 .

TOMASETTO, C., MOOG-LUTZ, C., REGNIER, C.H., SCHREIBER, V., BASSET, P. and RIO, M.C. (1995). Lasp-1 (MLN 50) defines a new LIM protein subfamily characterized by the association of LIM and SH3 domains. FEBS Lett373: 245 249.

WALLIN, J., WILTING, J., KOSEKI, H., FRITSCH, R., CHRIST, B. and BALLING, R. (1994). The role of Pax-1 in axial skeleton development. Development 120 1109-1121.

ZHAO, G.Q., ZHOU, X., EBERSPAECHER, H., SOLURSH, M. and DE CROMBRUGGHE, B. (1993). Cartilage homeoprotein 1, a homeoprotein selectively expressed in chondrocytes. Proc Natl Acad Sci USA 90: 8633-8637.

\section{Further Related Reading, published previously in the Int. J. Dev. Biol.}

See our recent Special Issue Fertilization, in honor of David L. Garbers and edited by Paul M. Wassarman and Victor D. Vacquier at: http://www.ijdb.ehu.es/web/contents.php?vol=52\&issue=5-6

Perichondrial-mediated TGF-beta regulation of cartilage growth in avian long bone development Marsha L. Crochiere, James K. Kubilus and Thomas F. Linsenmayer

Int. J. Dev. Biol. (2008) 52: 63-70

The retinoic acid metabolising gene, CYP26B1, patterns the cartilaginous cranial neural crest in zebrafish Susan Reijntjes, Adam Rodaway and Malcolm Maden Int. J. Dev. Biol. (2007) 51: 351-360

Fate of cranial neural crest cells during craniofacial development in endothelin-A receptor-deficient mice Makoto Abe, Louis-Bruno Ruest and David E. Clouthier Int. J. Dev. Biol. (2007) 51: 97-105

\section{A hypothesis linking low folate intake to neural tube defects due to failure of post-translation methylations of the cytoskeleton Natalie K. Björklund and Richard Gordon Int. J. Dev. Biol. (2006) 50: 135-141}

BMP4 promotes chondrocyte proliferation and hypertrophy in the endochondral cranial base

Lillian Shum, Xibin Wang, Alex A Kane and Glen H Nuckolls

Int. J. Dev. Biol. (2003) 47: 423-431

DIx5 regulates chondrocyte differentiation at multiple stages Andrew J Bendall, Gezhi Hu, Giovanni Levi and Cory Abate-Shen Int. J. Dev. Biol. (2003) 47: 335-344

Endochondral bone formation in toothless (osteopetrotic) rats: failures of chondrocyte patterning and type $\mathrm{X}$ collagen expression

S C Marks, C Lundmark, C Christersson, T Wurtz, P R Odgren, M F Seifert, C A Mackay, A Mason-Savas and S N Popoff Int. J. Dev. Biol. (2000) 44: 309-316

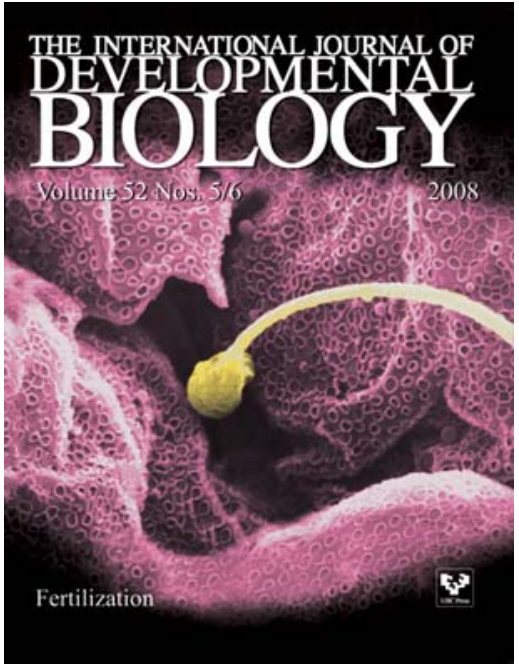

